\title{
QUINTAIS AGROFLORESTAIS COMO ESTRATÉGIA ALIMENTAR FAMILIAR NO ASSENTAMENTO 26 DE MARÇO, MARABÁ, PARÁ
}

\section{Regiane da Silva Miranda ${ }^{1}$; Jayane Santos Nunes²; Ilmaione Keiza de Souza Oliveira ${ }^{3}$; Dayanne Lustosa Mororó ${ }^{4}$; Rosana Quaresma Maneschy ${ }^{5}$.}

\author{
${ }^{1}$ Eng.Agrônoma, remirandaagro@hotmail.com \\ ${ }^{2}$ Eng.Agrônoma, jayzinhanunes@hotmail.com \\ ${ }^{3}$ Eng.Agrônoma, ilmaionekeiza@ hotmail.com \\ ${ }^{4}$ Eng.Agrônoma, dayanne_mba@hotmail.com \\ ${ }^{5}$ Professora Doutora da Faculdade de Ciências Agrárias de Marabá, Universidade Federal do Pará, \\ romaneschy@ufpa.br
}

\begin{abstract}
RESUMO: Os quintais agroflorestais (SAFQ) são sistemas de cultivo diversificados com espécies de múltiplos usos, envolvendo também a criação de pequenos animais. O objetivo deste estudo foi identificar a diversidade e função das espécies vegetais presentes nos quintais agroflorestais de unidades de produção familiares no assentamento rural "26 de Março", em Marabá - PA. A pesquisa foi realizada em junho de $2010 \mathrm{em}$ três estabelecimentos familiares. Utilizou-se a metodologia da pesquisa participante, entrevistas estruturadas com os membros da família e inventário de $100 \%$ das espécies vegetais da área do SAFQ. Os dados foram analisados através da estatística descritiva e para comparar a similaridade de espécies entre os quintais foi utilizado o índice de Sorensen. As frutíferas são predominantes nos sistemas avaliados, compondo aproximadamente 92\%; 86\% e 89\% das espécies presentes nos SAFQ1, SAFQ2 e SAFQ3, respectivamente. Foi observada similaridade média entre os quintais estudados. Esses agroecossistemas constituem-se numa alternativa viável de manejo racional devido sua composição florística, estrutura e possibilidade de produção diversificada auxiliando na segurança alimentar da unidade de produção familiar durante o ano.
\end{abstract}

PALAVRAS-CHAVE: agricultura familiar, diversidade, sistema agroflorestal.

\section{HOMEGARDENS FAMILY FOOD AS A STRATEGY IN SETTLEMENT OF MARCH 26, MARABÁ, PARA}

\begin{abstract}
The homegardens (AFSH) are diversified cropping systems with multiple uses, involving also the creation of small animals. The aim of this study was to identify the diversity and function of plant species found in homegardens of family production units in rural settlements "March 26" in Marabá - PA. The survey was conducted in June 2010 in three family farms. Used the methodology of participatory research, interviews with family members and inventory of $100 \%$ of plant species in AFSH area. Data were analyzed by descriptive statistics and to compare the similarity of species between the backyards was used by Sorensen index. The fruits are predominant in the systems evaluated, composing approximately $92 \%, 86 \%$ and $89 \%$ of species present in AFSH1, AFSH2 and AFSH3, respectively. Average was observed similarity between quintals studied. These agroecosystems have become a very viable alternative to rational management because its floristic composition, structure and the possibility of diversified production on food security of the farm family during the year.
\end{abstract}

KEY-WORDS: agroforesty system, diversity, family farm. 


\section{INTRODUÇÃO}

A insegurança alimentar configura-se como um problema para o país, inclusive na zona rural, em função do modelo de agricultura convencional extremamente excludente e conservador, baseado em poucas variedades de produtos voltados para o agronegócio Silva (2010). A agricultura familiar tem utilizado os sistemas agroflorestais (SAF) como estratégia para garantir o autoconsumo, fortalecendo as comunidades e diversificando os agroecossistemas.

Esses sistemas de uso da terra são considerados sustentáveis por possuírem características estruturais vantajosas, que são atribuídas às associações de espécies em estratos múltiplos, conservação do solo, reciclagem de nutrientes e a maior eficiência no seu uso, e o melhoramento do microclima, sobretudo para as espécies animais. Além de outros benefícios, tais como, eficiência de mão-de-obra, minimização do risco e oferta de diferentes produtos ao longo do ano (FERNÁNDEZ; NAIR, 1986).

Os SAF podem ser classificados de diversas formas, a mais utilizada é a estrutural com respeito à natureza do componente, categorizando-os em sistemas silviagrícolas, silvipastoris e agrossilvipastoris. Os silviagrícolas associam culturas anuais ao componente arbóreo, os silvipastoris são uma combinação entre árvores, pastagem e animais, enquanto os agrossilvipastoris além desses componentes integram também as culturas anuais (NAIR, 1991).

Os quintais agroflorestais, dentre os SAF, são os mais utilizados, pois são diversificados com espécies (agrícolas e florestais) de múltiplos usos, geralmente próximo a casa, envolvendo também a criação de pequenos animais. A produção obtida assegura algumas necessidades básicas, de agricultores familiares, como nutrição garantindo a segurança alimentar a partir da complementação da dieta básica diária com vitaminas e sais minerais em função do consumo de espécies frutíferas e saúde da família. Além disso, podem suprir a necessidade de lenha e madeira para a família e representam uma fonte adicional de renda a partir da venda do excedente da produção (MELÉNDEZ, 1996; VÍQUEZ et al., 1997).

Segundo Dubois et al. (1996) por esses sistemas integrarem diferentes componentes, em uma mesma área, têm como vantagens a capacidade de manter os níveis de produção melhorando a produtividade de forma sustentável. Pois são quase sempre manejados sem aplicação de agrotóxicos ou requerem quantidades mínimas dessas substâncias químicas, os efeitos negativos sobre o ambiente são, portanto, reduzidos. As raízes das árvores desempenharem um papel na aeração do solo, diminuído assim resistência à penetração em relação às monoculturas para a profundidade de $0-40 \mathrm{~cm}$, além de conservar a água por mais tempo no solo (CARVALHO; SOUZA, 2007). 
Assim, há uma boa utilização do espaço e do tempo pela alta biodiversidade presente com diferentes ciclos biológicos e formas de crescimento, sendo importante para o cotidiano do agricultor, pois fornece diversidade de alimentos e entradas, além de ser um bem valioso para programas futuros de cruzamento na forma de bancos de germoplasma, este último relevante para a sustentabilidade em longo prazo (TORQUEBIAU, 1992).

No Sudeste do Pará, mesorregião de grande concentração de assentamentos rurais muitas vezes criados em áreas ocupadas anteriormente por pastagens, faz-se necessário conhecer as estratégias utilizadas pelos agricultores para garantir a segurança alimentar das famílias. Esse estudo objetivou identificar a diversidade e função das espécies vegetais presentes nos quintais agroflorestais de unidades produção familiares no assentamento rural 26 de Março, em Marabá PA.

\section{MATERIAL E MÉTODOS}

A pesquisa foi realizada em junho de 2010 no Projeto de Assentamento (P. A.) 26 de Março, durante o Estágio de Campo obrigatório, do curso de Agronomia da Faculdade de Ciências Agrárias de Marabá.

O assentamento localiza-se na rodovia PA-150 a 25 km, município de Marabá, criado em dezembro de 2008. O clima é classificado como do tipo Afi no limite de transição para Awi e temperatura média de $28^{\circ} \mathrm{C}$. A cobertura vegetal predominante no assentamento são as pastagens. Antes da ocupação a área, denominada anteriormente de Fazenda Castanhal Cabaceira, era utilizada para a produção pecuária, tendo, portanto como cobertura vegetal predominante as pastagens (6.532,23 ha).

Apenas três das 12 famílias estudadas durante o estágio de campo possuíam quintal agroflorestal. Para seleção das famílias foram considerados os seguintes critérios: diversidade dos sistemas de produção, todos os membros da família deveriam aceitar e colaborar com a pesquisa e os lotes deveriam ser de fácil acesso.

A metodologia utilizada foi da pesquisa participante com a realização de entrevista estruturada com os membros da família utilizando um questionário com perguntas abertas e fechadas para a realização do levantamento da diversidade vegetal presente nos quintais de três unidades de produção e seus usos pelas famílias (medicinal, construção, móveis, ornamental, lenha, utensílios domésticos, sombra, experimentação).

Além da entrevista foi realizado o inventário de $100 \%$ da área do quintal. Os indivíduos vegetais identificados foram classificados quanto à ocorrência (espontâneo ou plantio) e quanto ao estádio de desenvolvimento (jovens, adultos e produtivos). As espécies arbóreas foram avaliadas quanto à altura total (AT), diâmetro 
a altura do peito (DAP) e diâmetro de copa (DC) dos indivíduos produtivos.

Os dados foram analisados através da estatística descritiva e para comparar a similaridade de espécies entre os quintais foi utilizado o índice de similaridade de Sorense, que é dado pela equação: IS $(\%)=(2 \mathrm{~A} / \mathrm{B}+\mathrm{C})$ $\mathrm{x} 100$, em que $\mathrm{A}=$ número de espécies comuns às duas áreas; $\mathrm{B}$ e $\mathrm{C}=$ número de espécies de cada uma das áreas (SORENSEN, 1972). A diversidade de espécie do sistema foi quantificada utilizando o índice de diversidade de Margalef (GLIESSMAN,
2005), obtido pela fórmula: Diversidade $=\mathrm{e}-$ $1 / \log \mathrm{n}$, onde $\mathrm{e}=$ número de espécies e $\mathrm{n}=$ número de indivíduos.

\section{RESULTADOS E DISCUSSÃO}

As unidades de produção familiares pesquisadas possuem área total de 45 a 50 ha, e o quintal ocupa de 0,23 a 0,50 ha sobre solo do tipo Podzólico Vermelho-Amarelo. A diversidade de espécies presentes nos quintais agroflorestais e seus diferentes usos por parte das famílias dos podem ser observados na Tabela 1.

Tabela 1. Espécies vegetais identificadas em quintais agroflorestais (SAFQ) de unidades de produção familiares e formas de utilização no P. A. 26 de Março, Marabá - PA

\begin{tabular}{|c|c|c|c|c|c|c|c|c|c|c|c|c|c|}
\hline \multirow{2}{*}{$\begin{array}{l}\text { FAMÍLIA/Nome científico } \\
\text { (nome comum) }\end{array}$} & \multirow[b]{2}{*}{ OCR } & \multirow[b]{2}{*}{ USO } & \multicolumn{3}{|c|}{ SAFQ1 } & \multicolumn{3}{|c|}{ SAFQ2 } & \multicolumn{2}{|c|}{ SAFQ3 } & \multirow[b]{2}{*}{$\mathrm{AT}(\mathrm{m})$} & \multirow{2}{*}{$\begin{array}{l}\text { DAP } \\
(\mathrm{cm})\end{array}$} & \multirow[b]{2}{*}{$\mathrm{DC}(\mathrm{m})$} \\
\hline & & & $\mathrm{J}$ & $\mathrm{A}$ & $\mathrm{P}$ & $\mathrm{J}$ & $\mathrm{A} \mathrm{I}$ & $\mathrm{P}$ & $\mathrm{J} \quad \mathrm{A}$ & $\mathrm{P}$ & & & \\
\hline $\begin{array}{l}\text { ANACARDIACEAE } \\
\text { Anacardium giganteum W. Hancock ex } \\
\text { Engl. (caju de janeiro) }\end{array}$ & PT & AL & & & & 3 & & & & & & & \\
\hline Anacardium ocidentale L. (cajú) & PT & $\mathrm{AL}$ & 2 & & & 1 & & & 3 & 6 & 2,9 & $4,0( \pm 5,5)$ & $3,0( \pm 0,08)$ \\
\hline Mangifera indica L. (manga) & PT & $\mathrm{AL}$ & 1 & & & & & 21 & 9 & & $2,5( \pm 0,5)$ & $5,0( \pm 6,9)$ & $2,0( \pm 0,8)$ \\
\hline Spondias dulcis Parkinson (cajá-manga) & $\mathrm{PT}$ & $\mathrm{AL} / \mathrm{SO}$ & & & & 1 & & & & & & & \\
\hline Spondias mombin L. (cajá) & PT & $\mathrm{AL}$ & 1 & & & & & & & & & 13,7 & 1,5 \\
\hline \multicolumn{14}{|l|}{ ANNONACEAE } \\
\hline Annona muricata L. (graviola) & PT & $\mathrm{AL}$ & & & & 1 & & & & & & & \\
\hline Rollinia mucosa (Jacq.) Baill (biriba) & PT & AL & & & & 1 & & & 3 & & $2,2( \pm 0,3)$ & $0,2( \pm 0,1)$ & $7,8( \pm 3,5)$ \\
\hline $\begin{array}{l}\text { ARAUCARICACEAE } \\
\text { Araucaria angustifolia (Bertol.) Kuntze } \\
\text { (pinheiro) }\end{array}$ & PT & SO & & & & 1 & & & & & & & \\
\hline \multicolumn{14}{|l|}{ ARECACEAE } \\
\hline Cocos nucifera (coco) & PT & $\mathrm{AL} / \mathrm{SO}$ & 6 & & & 10 & & & 3 & 1 & $1,4( \pm 0,2)$ & $4,7( \pm 6,5)$ & $3,9( \pm 1,4)$ \\
\hline Bactris gasipaes Kunth (pupunha) & $\mathrm{PT}$ & AL & & & & 12 & & & & & & & \\
\hline Euterpe oleracea Mart. (açaí) & PT & $\mathrm{AL}$ & & & & & & & 2 & & 0,5 & $0,1( \pm 0,1)$ & 1,0 \\
\hline Oenocarpus bacaba Mart. (bacaba) & PT & $\mathrm{AL}$ & & & & & & & & 1 & 5,0 & 0,3 & 6,0 \\
\hline \multicolumn{14}{|l|}{ BIXACEAE } \\
\hline Bixa orellana L. (urucum) & PT & $\mathrm{CON}$ & & & 1 & & & 3 & 1 & 1 & $3,2( \pm 0,2)$ & $3,2( \pm 4,4)$ & $3,5( \pm 0,4)$ \\
\hline \multicolumn{14}{|l|}{ CARICACEAE } \\
\hline Carica papaya (mamão). & PT & AL & & & 12 & & & 8 & 2 & 3 & $1,4( \pm 0,5)$ & $6,4( \pm 8,9)$ & $2,0( \pm 0,5)$ \\
\hline \multicolumn{14}{|l|}{ CECROPIACEAE } \\
\hline Cecropia hololeuca Miq. (embaúba) & & & & & & & & & 3 & & 5,0 & 0,1 & $4,2( \pm 0,6)$ \\
\hline \multicolumn{14}{|l|}{ FABACEAE } \\
\hline Cassia fastuosa Willd. (canafista) & PT & MAD & & & & & & & 1 & 1 & $3,2( \pm 1,8)$ & 0,2 & $7,5( \pm 0,7)$ \\
\hline
\end{tabular}




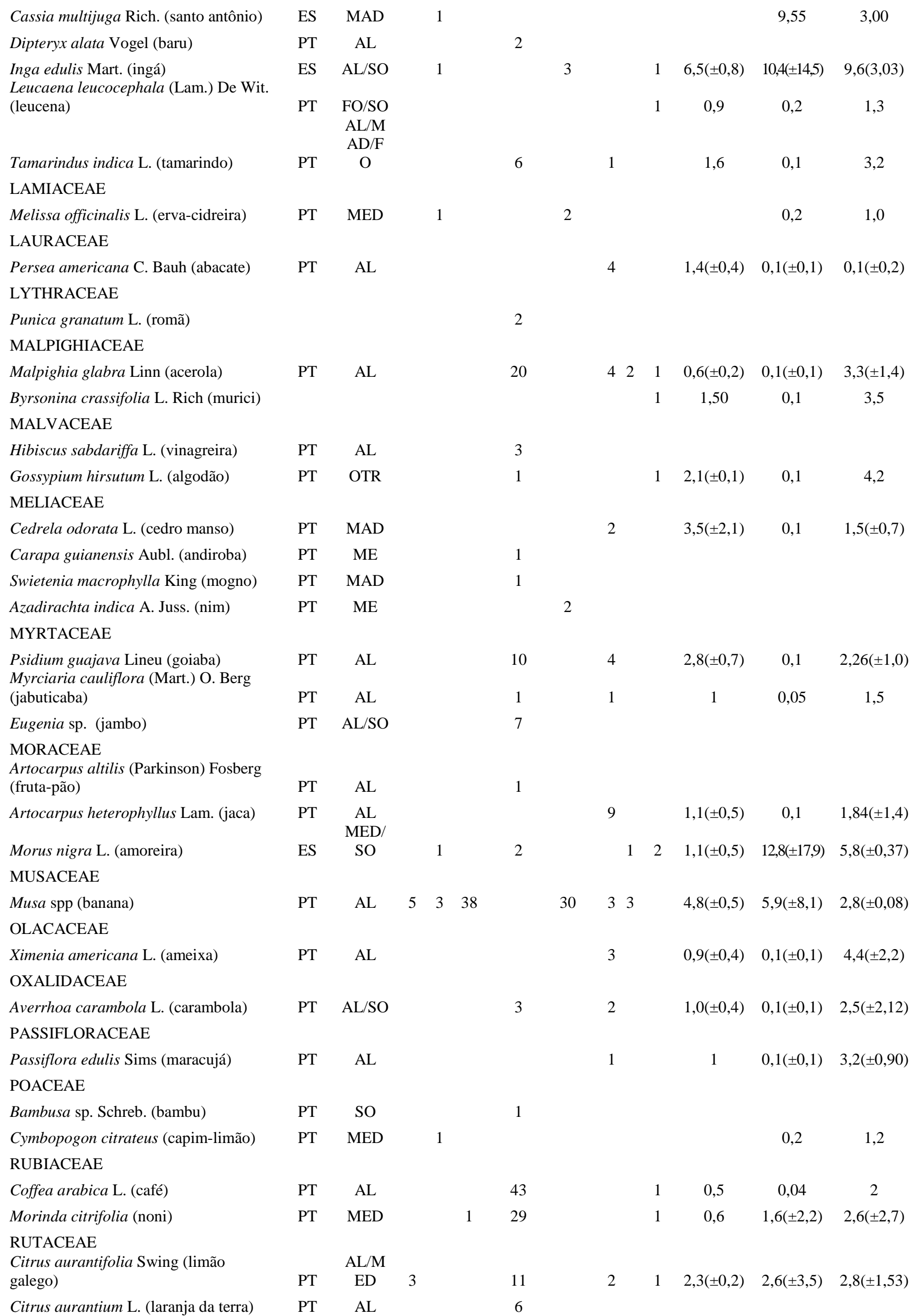




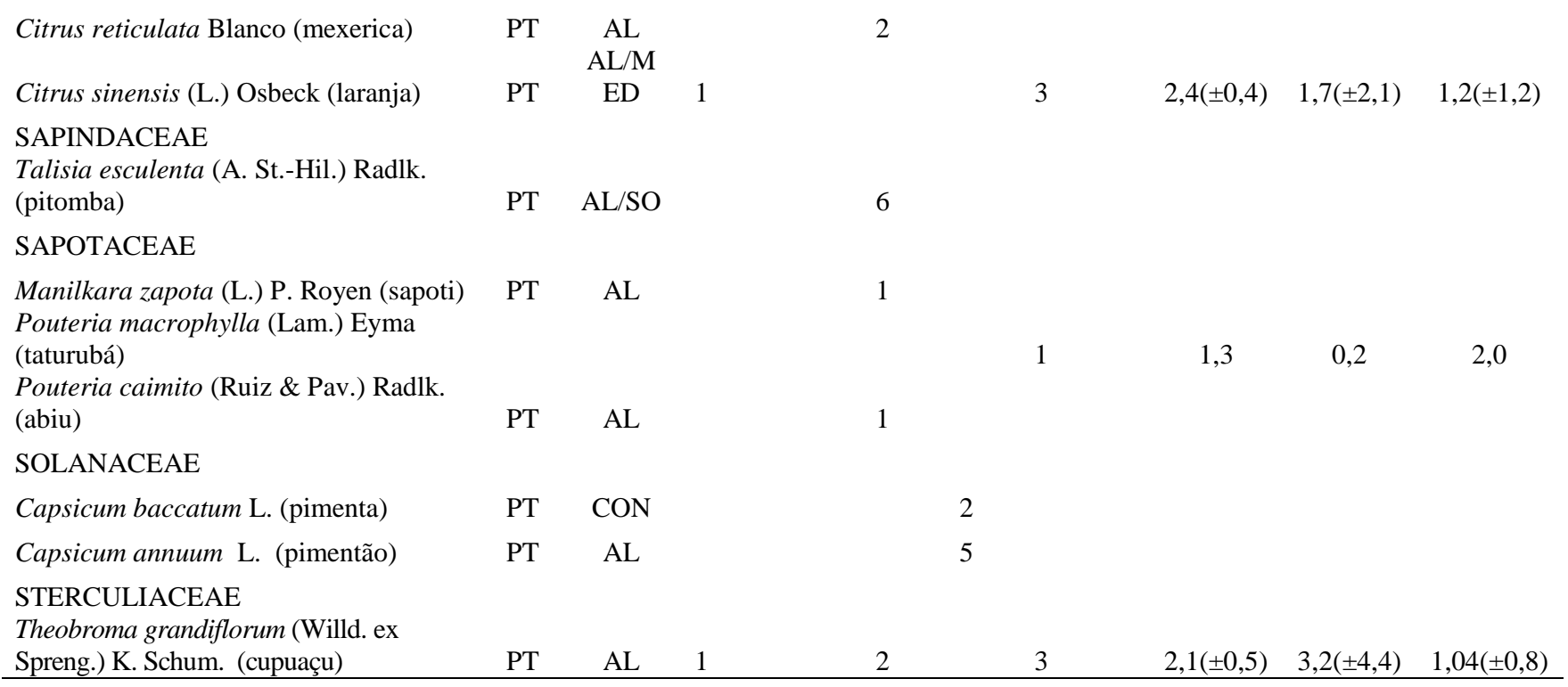

Onde: $\mathrm{OCR}=$ ocorrência, $\mathrm{PT}=$ plantio, $\mathrm{ES}=$ espontâneo, $\mathrm{AL}=$ Alimentação, $\mathrm{ME}=\mathrm{Medicinal}, \mathrm{CON}=$ Construção, $\mathrm{MO}=$ Móveis, $\mathrm{ORN}=$ Ornamental, $\mathrm{LEN}=$ Lenha, UDO= Utensílios domésticos, $\mathrm{SO}=$ Sombra, EXP= experimentação.

No SAFQ1 a maior parte das espécies vegetais foram introduzidas pelo agricultor e sua família (Tabela 1), uma vez que $90 \%$ da área da unidade de produção é ocupada por pastagem, já estabelecida antes da entrada da família na área. O arranjo das espécies se deu de forma aleatória a partir de um consórcio de banana e coco utilizando o espaçamento $3 \times 3$ m e $5 \times 5 \mathrm{~m}$, respectivamente. A família é constituída de seis membros que se alternam na divisão das tarefas de limpeza e manutenção do local. Sendo que o agricultor e a esposa são responsáveis pela escolha das espécies a serem implantadas no quintal. Caracterizando a existência de planejamento prévio para a distribuição espacial das plantas, construção de instalações para a criação dos animais (RONDÓN NETO et al., 2004) e execução dessas atividades que segundo Vieira et al. (2008), provavelmente, é favorecida pela localização dos quintais que se encontram às proximidades das casas, o que possibilita a participação de todos no trabalho.

As espécies frutíferas no SAFQ1 representam 92,2\% dos indivíduos presentes no quintal contribuindo para a alimentação da família e apenas $7 \%$ são de uso medicinal (capim limão, erva-cidreira e noni). Foi observado que o quintal está em processo de formação, pois a família está há dois anos no local, estando em fase produtiva apenas a bananeira, mamoeiro, noni e urucuzeiro.

A unidade de produção familiar do SAFQ2 é composta de $98 \%$ da sua área de floresta nativa, pois a família foi assentada na antiga área de reserva legal da fazenda Cabaceira. Apesar disso a família está investindo no estabelecimento do quintal, onde foi observado um número elevado de indivíduos na fase jovem e não produtivos esse fato se deve a família habitar apenas há 
dois anos na unidade de produção. A família do estabelecimento é composta por apenas um casal de idosos e a escolha das espécies vegetais para compor o quintal foi realizada por ambos. As tarefas relacionadas à manutenção do quintal são divididas entre o casal. A mulher realiza tarefas relacionadas aos cuidados da casa e coleta de produtos no quintal e o homem realiza trabalhos de limpeza, plantio e comercialização. Essas características descritas anteriormente são semelhantes a estudo realizado por Vieira et al. (2008) na mesorregião Nordeste do Pará.

As espécies frutíferas no SAFQ2 representam 85,8\%, cooperando para a alimentação da família, as de uso medicinais representam 12,7\% (Tabela 1), esses dados estão de acordo com Dubois et al. (1996), Ferreira e Pires-Sablayrolles (2009) que reportam que os quintais garantem a segurança alimentar da família, pois produzem alimento em quantidade e qualidade suficientes para manter a reprodutibilidade da família. Dentre as espécies vegetais levantadas no quintal verificaram-se que $23,5 \%$ das mesmas têm por finalidade proporcionar sombra; as espécies madeireiras, condimentares e outros usos correspondem a $0,38 \%$ cada.

O SAFQ2 esta em fase de desenvolvimento e a família pretende introduzir outras espécies com o intuito de comercializar o excedente da produção, pois atualmente o agricultor comercializa apenas a banana. Pode-se inferir que além do bem estar que os quintais proporcionam aos membros da família, a implantação desse sistema de cultivo auxilia na fixação do produtor no lote, uma vez que a família está investindo na introdução de espécies perenes.

$\mathrm{Na}$ unidade de produção do SAFQ3 repete-se a situação da propriedade do SAFQ1 onde as pastagens já predominavam antes da chegada da família. Que passou a investir na formação do SAFQ3, sobretudo com espécies frutíferas (89\%). Também possuem espécies madeireiras para construção de móveis e lenha (7\%), medicinais $(2 \%)$ e condimentares $(2 \%)$. As espécies que mais se destacaram foram: caju, jaca, manga, acerola e banana (Tabela 1). Os membros da família relataram a época de frutificação das espécies escolhidas para compor o quintal, sendo que a maior parte frutifica durante o ano todo (acerola, babaçu, café, coco, limão, noni e urucum). Carambola, cupuaçu, jaca e laranja, frutificam de novembro a dezembro. Enquanto que a manga frutifica de outubro a dezembro. Essa diversidade de espécies proporciona segurança alimentar para a família, pois os produtos do quintal complementam a dieta alimentar fornecendo sais minerais, carboidratos, proteínas, fibras e vitaminas (GAZEL FILHO, 2008).

Nos três quintais agroflorestais pesquisados verificou-se que as espécies frutíferas foram encontradas em maior frequência, por contribuírem na permanência do agricultor ao lote, além de colaborar com a segurança alimentar. Para Dubois et al. (1996) 
e Lunz (2007) a adoção de frutíferas em quintais agroflorestais também pode gerar lucros adicionais de renda, propiciar $\mathrm{o}$ aumento da biodiversidade e recuperar áreas degradadas e/ou abandonadas para que seja possível alcançar sustentabilidade nessas unidades de produção. Essa lógica está em consonância com o pensamento das famílias que planejam a ampliação da área do quintal e/ou introduzir animais domésticos e espécies vegetais (frutíferas e madeireiras) com o objetivo de complementar a dieta e a renda familiar a partir da comercialização da produção excedente. Que de acordo com Rosa et al. (2009) esse planejamento certamente deverá levar em conta disponibilidade de mão-de-obra familiar em função do calendário agrícola.

Com respeito à estrutura dos quintais agroflorestais, em geral as espécies foram introduzidas aleatoriamente, configurando o que Dubois et al. (1996) classificam como um SAF azonal. Seguindo o padrão típico de quintais tropicais, que segundo Nair (2004) apresenta em média três a seis estratos. $\mathrm{Na}$ Figura 1 foi exemplificada a distribuição dos indivíduos no SAFQ1. Na Figura 2 a estrutura vertical no estágio atual e em uma projeção de 25 anos para os três quintais agroflorestais avaliados.

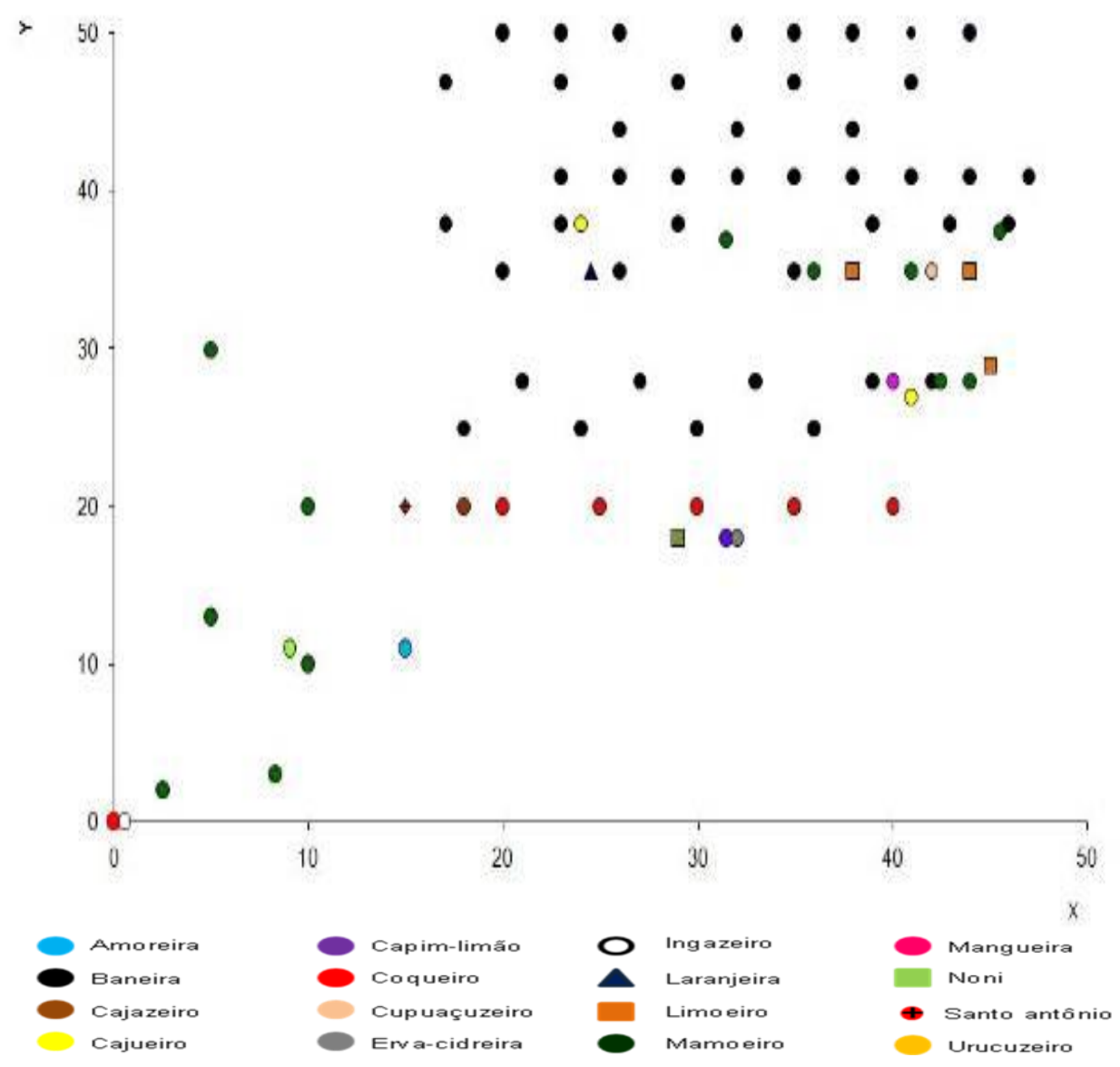

Figura 1. Esquema de localização das espécies vegetais identificadas na área do SAFQ1 em unidade de produção familiar, P. A. 26 de Março, Marabá - PA. 

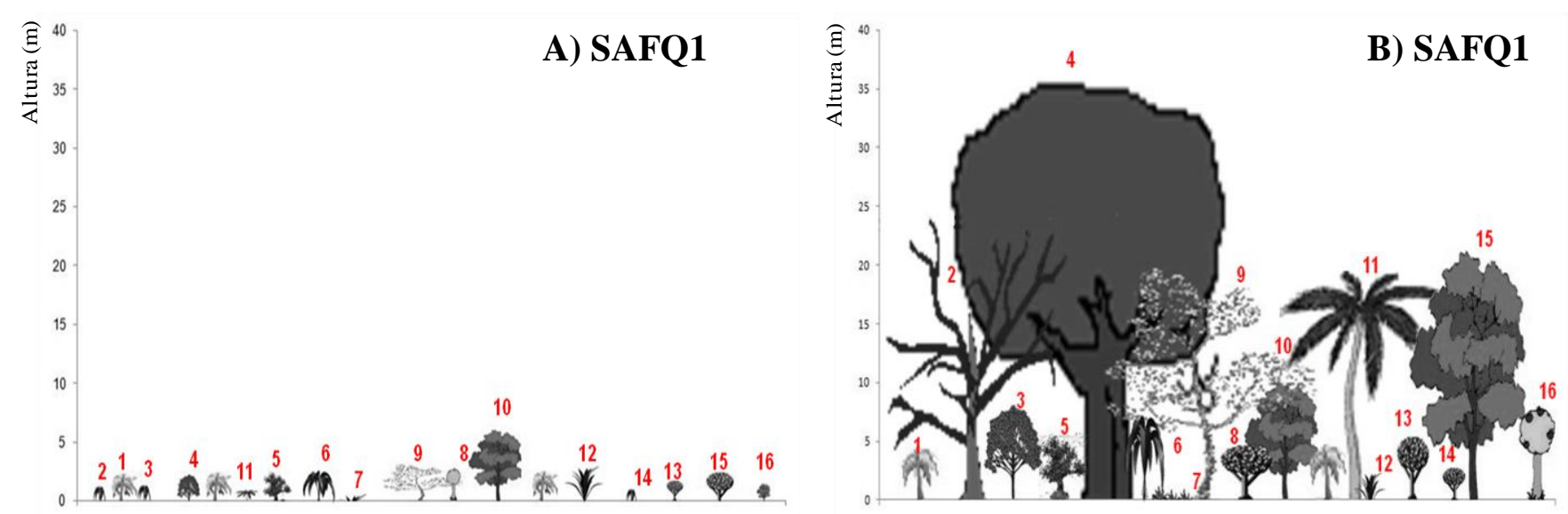

Onde: 1 - Bananeira, 2 - Cajazeiro, 3 - Cajueiro, 4 - Mangueira, 5 - Urucuzeiro, 6 - Mamoeiro, 7 - Erva-cidreira, 8 - Ingazeiro, 9 - Santo Antônio, 10 - Amoreira, 11 - Coqueiro, 12 - Capim-limão, 13 - Noni, 14 - Limoeiro, 15 - Cupuaçuzeiro, 16 - Laranjeira.
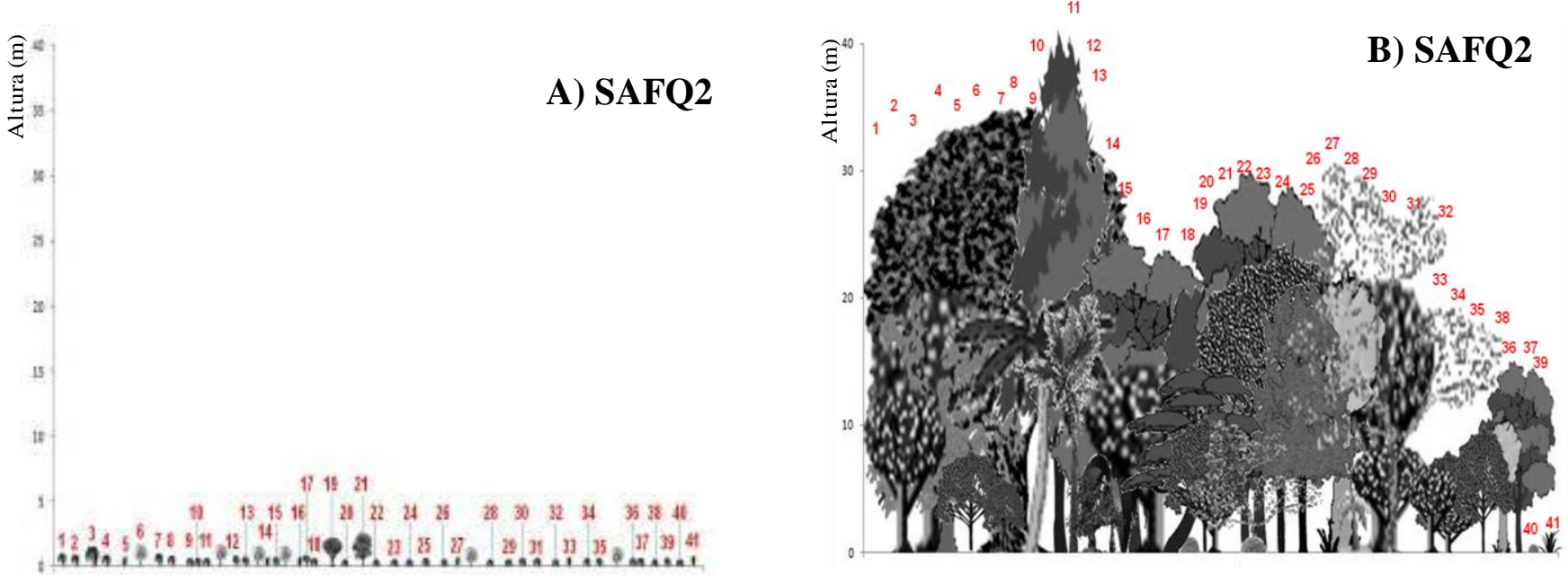

Onde: 1 - Cajazeira (cajá-manga), 2 - Caju de janeiro, 3 - Bananeira, 4 - Cajuí, 5 - Mangueira, 6 - Biribazeiro, 7 - Gravioleira, 8 - Pinheiro, 9 Coqueiro, 10 - Pupunheira, 11 - Urucuzeiro, 12 - Mamoeiro, 13 - Tamarindo, 14 - Baru, 15 - Erva Cidreira, 16 - Romanceira, 17 - Aceroleira, 18 - Algodoeiro, 19 - Goiabeira, 20 - Cupuaçuzeiro, 21 - Ingazeiro, 22 - Vinagreira, 23 - Andirobeira, 24 - Mogno, 25 - Nim, 26 - Fruta-pão, 27 - Jaqueira, 28 - Jabuticabeira, 29 - Jambeiro, 30 - Caramboleira, 31 - Bambueiro, 32 - Cafeeiro, 33 - Noni, 34 -Laranja da terra, 35 Limoeiro, 36 - Tangerineira (mexerica), 37 - Pitombeira, 38 - Abiu, 39 - Sapoti, 40 - Pimenteira e 41 - Pimentão.
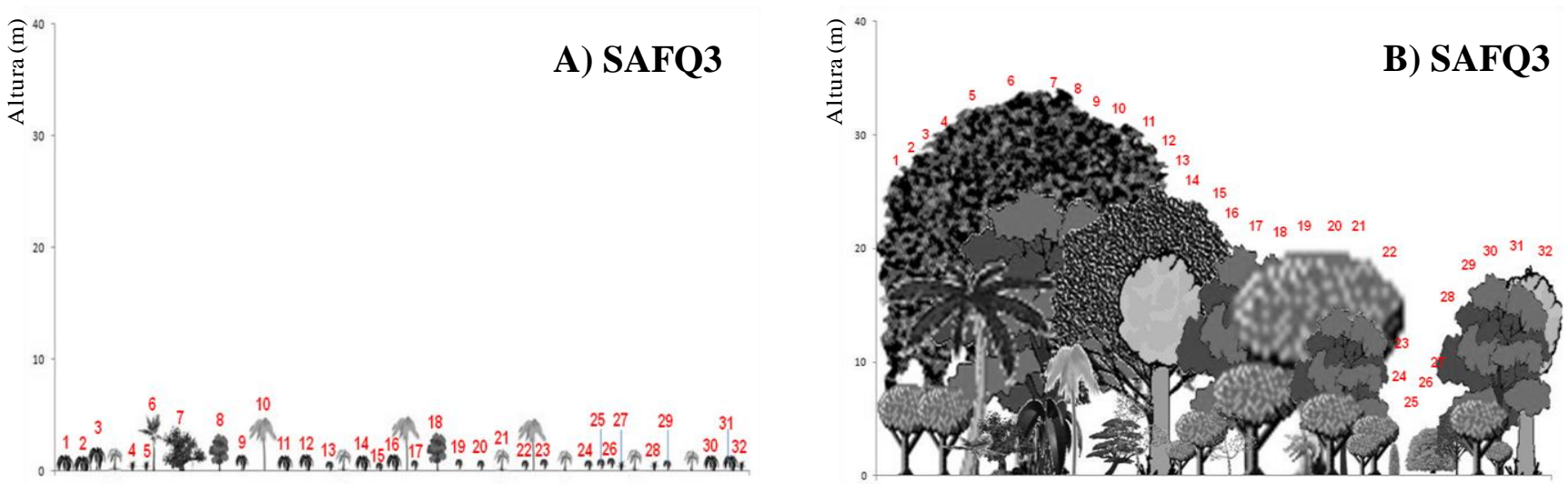

Onde: 1 = Cajueiro, $2=$ Mangueira, $3=$ Biribazeiro, $4=$ Coqueiro, $5=$ Açaizeiro, $6=$ Bacaba, $7=$ Urucuzeiro, $8=$ Canafista, $9=$ Mamoeiro, 10 = Embaúba, $11=$ Tamarindo, $12=$ Ingazeiro, $13=$ Leucena, $14=$ Abacateiro, $15=$ Acerola, $16=$ Muruci, $17=$ Algodoeiro, $18=$ Cedro manso, $19=$ Amoreira, $20=$ Jaqueira, $21=$ Bananeira, $22=$ Goiabeira, $23=$ Jambeiro, $24=$ Ameixa, $25=$ Caramboleira, $26=$ Maracujazeiro, 27 = Cafeeiro, $28=$ Noni, $29=$ Laranjeira, 30 = Limoeiro, $31=$ Tuturubá, $32=$ Cupuaçuzeiro.

Figura 2. Perfil da estrutura vertical dos SAFQ1, SAFQ2 e SAFQ3 de unidades de produção familiares, P. A. 26 de Março, Marabá - PA, A) Estágio atual; B) Projeção para 25 anos. 
Em relação ao índice de Sorense, os quintais agroflorestais do P. A. 26 de Março obteve a seguinte sequência de similaridade:
SAFQ3 x SAFQ2 > SAFQ3 x SAFQ1 > SAFQ2 x SAFQ1 (Quadro 1).

Quadro 1. Índice de similaridade de Sorense (\%) em quintais agroflorestais (SAFQ) de unidades de produção familiares no P. A. 26 de Março, Marabá - PA.

\begin{tabular}{|c|c|c|}
\hline & SAFQ1 & SAFQ2 \\
\hline SAFQ3 & 50,00 & 52,05 \\
\hline SAFQ2 & 42,11 & \\
\hline
\end{tabular}

A similaridade entre os quintais foi considerada média. Isso pode ser explicado em função da preferência das famílias por espécies em função do uso (frutífera, madeireira e medicinal), disponibilidade de material de propagação vegetal, adaptabilidade da espécie, demanda de mão de obra relacionada a tratos culturais específicos. Segundo Pinto-Coelho (2000) a frequência de determinadas espécies no quintal agroflorestal está relacionada com sua função seccional, além da preferência do agricultor pelo potencial de aproveitamento da espécie, assim como pela disponibilidade de material para reprodução.

A riqueza da diversidade de espécies vegetais identificada nos quintais estudados foi alta (Tabela 2), considerando que em monocultura o índice de Margalef é zero segundo Gliessman (2005). O fato dos quintais do P. A. 26 de Março serem recentes e das famílias ainda estarem experimentando esse sistema de cultivo, bem como, a associação das diferentes espécies identificadas pode estar colaborando para uma riqueza maior do que as relatadas anteriormente em sistemas agroflorestais (SAF) já estabelecidos em unidades de produção de assentamentos rurais familiares no Sudeste do Pará. Como por exemplo, em parcelas de SAF comerciais em que o índice de Margalef variou de 1,02 até 1,27 (FEITOSA, 2011) e em quintais de mais de 10 anos de formação em que a variação do índice foi de 6,26 até 14,13 (PEREIRA, 2011).

Tabela 2. Medidas da diversidade de espécies vegetais em quitais agroflorestais (SAFQ) de três unidades de produção familiares, P. A. 26 de Março, Marabá - PA.

\begin{tabular}{lccc}
\hline & SAFQ1 & SAFQ2 & SAFQ3 \\
\hline Número de espécies (s) & 16 & 41 & 32 \\
Número de indivíduos (N) & 80 & 268 & 99 \\
Relação N/s & 5 & 6,5 & 3,1 \\
Riqueza relativa de espécies* & alta & alta & alta \\
Uniformidade relativa de espécies** & baixa & baixa & baixa \\
Índice de Margalef & 15,5 & 40,6 & 31,5 \\
\hline
\end{tabular}

*Em monocultura é considerada baixa. ** Em monocultura é considerada alta (GLIESSMAN, 2005). 
A diversidade de espécies nativas identificadas nos quintais quando por meio de um plano de manejo adequado, podem se constituir de acordo com Florentino et al. (2007) em uma alternativa, de baixo custo, para a conservação da biodiversidade local e o conhecimento tradicional a elas associado (FERREIRA; PIRES-SABLAYROLLES, 2009).

Unidades de produção familiares que se constituem de SAFs tradicionais como os quintais agroflorestais e seus outros subsistemas diversificados da área de estudo, segundo Castro et al. (2009) podem garantir a sustentabilidade socioeconômica de uma localidade, configurando-se em uma melhor alternativa desde que adaptada às condições locais.

\section{CONCLUSÃO}

Foi identificada maior frequência de espécies frutíferas com finalidade alimentar possibilitando uma produção diversificada durante o ano e auxiliando na segurança alimentar das famílias.

A similaridade entre os quintais foi considerada média e diversidade de espécies alta constituindo agroecossistemas de composição florística e estrutural diretamente relacionada à funcionalidade das espécies para as famílias.

\section{REFERÊNCIAS}

DUBOIS, J. C. L.; VIANA, V. M.; ANDERSON, A. B. Manual Agroflorestal para a Amazônia. Rio de Janeiro: REBRAF, 1996. 228 p.

CARVALHO, A. J. A.; SOUZA, E. H. Caracterização física dos solos dos quintais agroflorestais e cultivos monotípicos na região de Amargosa, Bahia. Revista Brasileira de Agroecologia, v. 2, n. 2, p. 941-944, out. 2007.

CASTRO, A. P. DE; FRAXE, T. DE J. P.; SANTIAGO, J. L.; MATOS, R. B.; PINTO, I.

C. Os sistemas agroflorestais como alternativa de sustentabilidade em ecossistemas de várzea no Amazonas. Acta Amazonica, v. 39, n. 2, p. 279-288, 2009.

FEITOSA, L. L. Avaliação agronômica de parcelas de sistemas agroflorestais implantadas no município de Eldorado dos Carajás, Pará. Marabá, 2011, 139 f. Trabalho de conclusão de curso (Agronomia)Faculdade de Ciências Agrárias de Marabá, Universidade Federal do Pará, Marabá, 2011.

FERNÁNDEZ, E. C. M.; NAIR, P. K. R. An evaluation of the structure and function of tropical homegardens. Agricultural Systems, v. 2, p.73-86, 1986.

FERREIRA, T. $\quad$ B.; PIRESSABLAYROLLES, M. G. Quintais Agroflorestais como Fontes de Saúde: plantas medicinais na Comunidade de Vila Franca, Reserva Extrativista Tapajós-Arapiuns, Pará. Revista Brasileira de Agroecologia, v. 4, n. 2, 2009. 
FLORENTINO, A. T. N.; ARAÚJO, E. DE L.; ALBUQUERQUE, U. P. Contribuição de quintais agroflorestais na conservação de plantas da Caatinga, Município de Caruaru, PE, Brasil. Acta Botanica Brasilica, v. 21, n. 1, p. 37-47, 2007.

GAZEL FILHO, A. B. Composição, Estrutura e Função de Quintais Agroflorestais no Município de Mazagão, Amapá. Belém: UFRA, 2008. 104p. Tese de Doutorado.

GLIESSMAN, S. R. Agroecologia: processos ecológicos em agricultura sustentável. Porto Alegre: UFRGS, 2005. 653 p.

LUNZ, A. M. P. Quintais agroflorestais e o cultivo de espécies frutíferas na Amazônia. Revista Brasileira de Agroecologia, v. 2, n. 2, p. 1255 - 1258, out. 2007.

MELÉNDEZ, L. Estrategia para el establecimiento de huertos caseros en asentamientos campesinos en el area de conservación de Tortuguero, Costa Rica. Agroforesteria em las Américas, v. 9, n.3, p. 25-28, 1996.

NAIR, P. K. P. The enigma of tropical homengardens. Agroflorestry Systems. v. 61, p. 135-152, 2004.

NAIR, P. K. R. State of the art of agroforestry systems. Forest Ecology and Management, v. 45, p. 5-29, 1991.
PEREIRA, C. N. Quintais agroflorestais no projeto de assentamento Belo Horizonte, São Domingos do Araguaia, Pará. Marabá, 2011, 49 f. Trabalho de conclusão de curso (Agronomia)- Faculdade de Ciências Agrárias de Marabá, Universidade Federal do Pará, Marabá, 2011.

PINTO-COELHO, R. M. Biodiversidade: métodos de mensuração. In: _ Fundamentos em Ecologia. Porto Alegre: Artmed, p. 81-91, 2000.

RONDON NETO, R. M.; BYCZKOVSKI, A.; WINNICKI, J. A.; SIMÃO, S. M. M.; PASQUALOTTO, T. C. Os quintais agroflorestais do assentamento rural Rio da Areia, município de Teixeira Soares, PR. Cerne, Lavras, v. 10, n. 1, p. 125-135, jan./jun. 2004.

ROSA, L. DOS S.; VIEIRA, T. A.; PIRES, H. C. G. Quintais agroflorestais em comunidades rurais de Bonito, Pará. Revista Brasileira de Agroecologia, Porto Alegre, v 4,n 2, 2009.

SILVA, J. S. Agroecologia: base estratégica para a segurança alimentar. Revista Verde de Agroecologia e Desenvolvimento Sustentável, v.5, n.1, p. 1 - 6, jan./mar. 2010.

SORENSEN, T. A method of stablishing groups of equal amplitude in plant society based on similarity of species content. In: Odun, E. P. (ed.). Ecologia. 3 ed. México: Interamericana, p. 640, 1972. 
TORQUEBIAU, E. Are tropical agroforestry home gardens sustainable? Agricultura, Ecosystems and Environment, v.41, p. 189207, 1992.

VIEIRA, T. A.; ROSA, S. L.; MODESTO, S. R.; SANTOS, M. M. Gêneros e sistemas agroflorestais: o caso Igarapé-Açu Pará,
Brasil. Revista de Ciências Agrárias, Belém, n. 50, p. 143-154, 2008.

VÍQUEZ, E.; PRADO, A.; OÑORO, P. Caracterización del huerto mixto tropical " $\mathrm{La}$ Asunción”, Masatepe, Nicaragua. Agroforesteria em las Américas, Turrialba, n. 2, p. 5-9, 1994. 